\title{
SY48-3 Symposium
}

\section{Pharmacogenomic Implications of Population Diversity and Admixture}

\section{Guilherme Suarez-Kurtz}

\section{Department of Pharmacology, National Cancer Institute, Brazil}

Pharmacogenetics/pharmacogenomics relies on human genetic diversity. My talk will initially focus on the pharmacogenomic consequences of human demographic history and genetic diversity. I will then move to the more formal pharmacogenetic arena and discuss the impact of population diversity on the worldwide distribution of common variants in pharmacogenes, using the NAT2 gene as an example. Inference of NAT2 phenotypes across global populations by a tag SNP or combinations of $2-4$ SNPs will be contrasted with the standard 7-SNP panel. Next, the pharmacogenomic implications of population admixture will be examined, drawing data from the two most populous countries in Latin America, i.e. Brazil and Mexico, where a kaleidoscopic combination of individual proportions of Native American, European and sub-Saharan African ancestries prevails. Accordingly, average admixture proportions are not predictive of the corresponding proportions at the individual level. Thus, ethnic/race/Color categories cannot capture the complex structure of Latin American peoples. As a consequence of admixture, the genetic differentiation of common pharmacogenetic variants among Brazilians is much attenuated in comparison to their relevant ancestral populations, especially Europeans and Africans. The impact of such attenuation on the predictive performance of warfarin dosing algorithms in Brazilians, compared to their ancestral proxies, will be discussed. Studies in admixed populations have the potential to reveal novel pharmacogenomic associations: a distinct example is the disclosure of gene-dose effect of CYP3A5 polymorphisms on the dose requirement of tacrolimus, first reported in Brazilian renal transplant patients. Pharmacogenomic implementation in clinical practice must take into account the abundance of rare variants (minor allele frequency $<1 \%$ or $<0.5 \%$ ) in our genome. Due to their recent origin there is very little sharing of rare variants between continental populations or within continental populations. A substantial effort will be required to catalogue rare variants and to develop reliable algorithms to identify their putative functional effects and potential clinical relevance. 